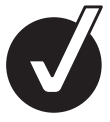

EDITOR'S

CHOICE

\title{
Callers' experiences of making emergency calls at the onset of acute stroke: a qualitative study
}

\author{
Stephanie P Jones, ${ }^{1}$ Hazel A Dickinson, ${ }^{2}$ Gary A Ford, ${ }^{3}$ Josephine M E Gibson, ${ }^{1}$ \\ Michael J Leathley, ${ }^{1}$ Joanna J McAdam, ${ }^{1}$ Alison McLoughlin, ${ }^{2}$ Tom Quinn, ${ }^{4}$ \\ Caroline $L$ Watkins, ${ }^{1}$ on behalf of the Emergency Stroke Calls: Obtaining Rapid \\ Telephone Triage Group
}

\begin{abstract}
- An additional appendix is published online only. To view this file please visit the journal online (http://emj.bmj.com/ content/29/6.toc)
\end{abstract}

${ }^{1}$ Clinical Practice Research Unit, School of health, University of Central Lancashire, Preston, UK ${ }^{2}$ Lancashire Teaching Hospitals NHS Foundation Trust, Preston, UK

${ }^{3}$ Royal Victoria Infirmary,

Newcastle, UK

${ }^{4}$ Division of Health and Social Care, University of Surrey, Guildford, UK

\section{Correspondence to} Stephanie P Jones, Clinical Practice Research Unit, University of Central Lancashire, Brook 425, School of Nursing and Caring Sciences, Preston PR1 2HE, UK;

sjones10@uclan.ac.uk

Accepted 9 May 2011 Published Online First 8 July 2011

\section{ABSTRACT \\ Background Rapid access to emergency medical} services (EMS) is essential at the onset of acute stroke, but significant delays in contacting EMS often occur. Objective To explore factors that influence the caller's decision to contact EMS at the onset of stroke, and the caller's experiences of the call.

Methods Participants were identified through a purposive sample of admissions to two hospitals via ambulance with suspected stroke. Participants were interviewed using open-ended questions and content analysis was undertaken.

Results 50 participants were recruited (median age 62 years, $68 \%$ female). Only one of the callers (2\%) was the patient. Two themes were identified that influenced the initial decision to contact EMS at the onset of stroke: perceived seriousness, and receipt of lay or professional advice. Two themes were identified in relation to the communication between the caller and the call handler: symptom description by the caller, and emotional response to onset of stroke symptoms.

Conclusions Many callers seek lay or professional advice prior to contacting EMS and some believe that the onset of acute stroke symptoms does not warrant an immediate 999 call. More public education is needed to improve awareness of stroke and the need for an urgent response.

\section{INTRODUCTION \\ Background}

Stroke is a leading cause of mortality and disability worldwide. ${ }^{1}$ Rapid access to emergency stroke care can reduce death and dependency by enabling immediate provision of interventions such as physiological monitoring and stabilisation and thrombolysis. ${ }^{2}$

\section{Importance}

Up to $70 \%$ of all stroke patients obtain first medical contact from the Emergency Medical Service (EMS). ${ }^{3-5}$ EMS call handlers in the UK currently use the Advanced Medical Priority Dispatch System (AMPDS) to categorise ambulance response and decide on the level of medical care sent. Although this system is effective at ruling out acute stroke in people with other conditions, it is poor at correctly identifying acute stroke, with over $50 \%$ of strokes being misclassified. ${ }^{6}$ The communication between the caller and emergency call handler is crucial in identifying suspected stroke, minimising delays and improving outcomes. While problems with communication have been previously identified, ${ }^{7}$ no studies have explored the caller's experience of making a 999 call at the onset of stroke symptoms.

\section{Purpose of investigation}

The purpose of the study was to identify the features that expedited or delayed people's initial decision to contact EMS at the onset of stroke, and to explore callers' experiences of the call.

\section{METHODS}

\section{Study design}

This was a qualitative study using semi-structured interviews and content analysis.

\section{Setting}

The study was conducted in two hospitals in northwest England (total population approximately 700000 ) serving approximately 1600 new stroke patients each year.

\section{Selection of participants}

A criterion based purposive sample ${ }^{8}$ was identified. Subjects were selected if they were admitted to hospital with a diagnosis of acute stroke and arrived via ambulance after contacting EMS (calling 999) or via a personal medical alert system, during a 3-month period (10 October 2008-22 January 2009). Subjects were excluded if their admission was arranged by primary care (family physician), they attended the emergency department directly or they had a stroke while in hospital. Relatives or carers of patients who had died or remained critically ill were not approached. The person who had made the initial call to EMS (999 call) after the onset of stroke symptoms, was identified as the potential participant in the study, whether the patient, a relative or another person.

\section{Procedure}

Patients (or next of kin if the patient had significant cognitive impairment or communication problems) were initially approached during their inpatient stay and within a maximum of 2 weeks after admission, by a member of the clinical team. Participants were given $24 \mathrm{~h}$ to decide if they would like to participate. Written informed consent was obtained. The interviews were conducted face to face, at either the stroke unit or the caller's home. Interviews were digitally recorded. 


\section{Data collection}

An interview schedule was initially developed by the authors and was piloted with input from patient representatives to ensure clarity and validity, resulting in a final interview guide of 19 questions.

\section{Primary data analysis}

Interview recordings were initially transcribed verbatim. Analysis was undertaken using a constant comparative method in order to identify patterns and relationships within the data. ${ }^{9}$ Open codes were created for each interview, and were then clustered to each other in order to create broader categories. These categories were grouped to develop themes. Each interview was analysed independently by two of the three researchers (SPJ, JJMcA, JMEG); discrepancies were resolved by discussion with the third researcher. The research team also met regularly to discuss emerging themes in order to ensure a consistent approach to data collection and coding. The themes were reviewed by patient representatives.

\section{RESULTS}

Table 1 outlines the characteristics of the callers who participated, their relationship to the patient, and action taken.

Four central themes were identified. Two of these related to the initial decision to contact EMS at the onset of stroke: perceived seriousness; and seeking and receiving lay or professional advice. Two themes related to communication between the caller and the call handler: description of stroke symptoms by the caller; and emotional response to acute stroke symptoms. Verbatim quotes in the following section are followed by the caller's relationship to the patient, gender and age of the caller.

\section{Perceived seriousness}

Twenty-five $(50 \%)$ callers recognised that the symptoms were stroke-related and serious, and contacted EMS immediately. They based this on previous experience of stroke $(n=13)$, knowledge $(n=6)$ or a combination of both $(n=6)$.

A further nine (18\%) callers also recognised that the symptoms were stroke-related, but were unsure of the seriousness, seeking further advice before contacting EMS. These callers either contacted a family member $(n=4)$ or the family physician $(\mathrm{n}=5)$ for support and advice before calling 999.

"I rung the (family physician) surgery and the doctor said,
'ambulance straightaway'." Friend, male, 69.

The remaining $16(32 \%)$ callers did not recognise the symptoms as stroke. Five (10\%) of these callers recognised that

Table 1 Characteristics of participants

\begin{tabular}{ll}
\hline Characteristic & Participants $(\mathbf{n}=\mathbf{5 0})$ \\
\hline Median age (years) & 62 \\
Age range (years) & $24-83$ \\
Female (\%) & 68 \\
Caller & \\
$\quad$ Patient & 1 \\
$\quad$ Relative/friend/neighbour & 41 \\
Other bystander & 6 \\
Personal medical alert system & 2 \\
Action $(\mathbf{n})^{*}$ & \\
Called EMS immediately & 30 \\
Delayed contacting EMS & 20 \\
\hline
\end{tabular}

*See appendix 1 for detail of actions taken.

EMS, emergency medical services. something was sufficiently serious to call 999 immediately. Their concerns included suspected heart attack and lack of movement. Nine (18\%) callers were unsure of the seriousness of the situation and either delayed calling $999(n=2)$, or sought further lay advice $(n=4)$ or professional advice from the family physician ( $\mathrm{n}=3$ ) before calling 999.

"I didn't think it was that serious to call the ambulance but then when I explained the symptoms to (daughter) she said well better call the ambulance." Husband, 66.

Two callers (4\%) recognised that the situation was serious but still sought further advice before calling 999. One of these callers contacted NHS Direct (a nurse led telephone helpline) because they did not want to waste the emergency services' time, and the other contacted a relative and subsequently their family physician.

\section{Seeking and receiving lay or professional advice}

Eighteen (36\%) callers who were unsure about the significance of the symptoms often sought advice and clarification from others before dialling 999. Sources of advice included friends or relatives $(n=9)$, of whom two asked for further advice from the family physician or the emergency department, primary care services $(n=8)$ and NHS Direct $(n=1)$. Up to three steps were taken before eventually calling 999 (online appendix 1), with the potential to introduce delay with each extra step.

Although participants recognised that they were delaying emergency help by seeking advice from sources other than EMS, they often tried to justify this delay.

"Rather than calling the emergency services out I just wanted to wait that few minutes or get somebody else to tell me that was, you know, get a second opinion." Husband, 66.

Of the eight (16\%) callers who sought help from primary care, seven received telephone advice to contact EMS immediately.

"So first of all I rang actually Primary Care (family physician), and once I gave them the symptoms, they said just ring 999 immediately, which I did." Wife, 45.

One caller made an appointment for the patient to see the family physician. Following deterioration of their condition and a second phone call to primary care, they were advised to call 999 immediately.

\section{Description of stroke symptoms by the caller}

Most callers ( $n=34,68 \%$ ) stated during the interview that they had suspected that the person was having a stroke, but only 27 (54\%) had mentioned the word 'stroke' to the call handler.

"I said that she has fallen but it looks a bit, her mouth's funny, I didn't use the word stroke, I said her mouth's funny and her arm's weird." Daughter, 56.

Of the 27 callers who mentioned 'stroke' to the call handler, 24 also described other stroke-specific symptoms. These included movement problems or numbness on one side $(n=19)$, altered speech $(n=16)$, facial droop $(n=11)$ and a fall $(n=6)$.

"I think he's had a stroke...his mouth's drooped to one side, he's slurring his speech and he can't move one side of his body." Neighbour, male, 45.

Less stroke-specific descriptors such as headache, collapse, funny turn, unable to get out of bed and unresponsive were also mentioned. 
Five $(10 \%)$ callers had reported conditions other than stroke such as heart attack, and 18 (36\%) were unsure of what they had reported to the call handler.

\section{Callers' emotional response to acute stroke symptoms}

Twenty-six $(52 \%)$ callers described their emotional response to the onset of stroke, including feelings of panic $(n=7)$, nervousness $(n=4)$ and fear $(n=3)$. Nine callers described feelings of frustration, worry and upset. Three callers described the need to overcome their emotions and to stay calm.

"You know nothing about it, you start panicking." Husband, 76.

Callers recognised that there was a potential conflict for the call handler between dealing efficiently with the call and with the caller's emotional distress.

\footnotetext{
"You have to explain everything, you know, what's going on, her age, date of birth, address, name...but you're panicking thinking please don't just ask these questions, get here straight away." Daughter-in-law, 45.
}

However, the call handler's efficient manner was supportive, and helped the caller to stay calm. Some callers also felt that the call handler gave emotional support in addition to practical advice.

"Yeah she was very reassuring with her tone of voice...you know, managed to keep me calm because normally I start panicking a bit." Wife, 50

It was also identified that once the call was made, the call handler took responsibility for the situation.

"She does calm you down and help you cope with the situation, you feel like you're just going with the flow." Wife, 69 .

Callers found it helpful to be assured that the ambulance was on its way throughout the call.

"They were speaking to me all the time, telling me the emergency people were on their way now...it gives you confidence, it does really." Husband, 76.

However, it was also felt that the call handler's assurance that the ambulance was en route was not always clear.

"I don't recall her actually saying an ambulance is on the way...they ask you all these questions and you're thinking... have you decided yet, are you sending one?" District nurse, female, 33.

\section{DISCUSSION}

Thirty participants sought first medical contact from the EMS, with the remainder contacting primary medical services or a relative or friend. Only one patient (2\%) called an ambulance themselves, consistent with previous studies. ${ }^{10}{ }^{11}$ Patients who are alone often contacted a family member. ${ }^{12}$ Nine participants $(18 \%)$ in our study contacted a family member or friend for help.

People's emotional response to the onset of stroke symptoms was a theme that emerged during data collection, with $52 \%$ of callers describing feelings such as panic, nervousness and fear. Participants contacted primary care, family or friends in order to receive advice or support about the course of action that should be taken. This was a common cause of delay in contacting EMS.

The efficiency of the call handler was found to be reassuring. Emotional support and practical advice were both important to callers. However, some callers were unsure as to whether an ambulance had actually been dispatched during the call.
Although $68 \%$ of the callers suspected stroke, only $54 \%$ of the sample reported this to the call handler, consistent with previous findings that stroke is reported as the presenting problem in $44 \%$ of ambulance calls leading to a final diagnosis of acute stroke. ${ }^{11}$ Currently, AMPDS algorithms require the call handler to ask questions about 'what has happened' to the patient, rather than asking what the caller suspects may be the diagnosis. This may make it more difficult for callers to report their suspicion of stroke.

Previous research into decision making processes in people with symptoms of myocardial infarction has identified that prior knowledge of symptoms alone is not enough to initiate prompt action. ${ }^{13}$ A recent review of the public's awareness of stroke found that although $27-100 \%$ of participants stated that they would call the EMS, only $18 \%$ had actually done so. ${ }^{14}$ Emotional response and context appear to be influential in deciding to seek emergency help. ${ }^{13}$

This is the first study to examine in detail the factors that influence the initial decision to contact EMS at the onset of stroke symptoms, and to explore the stroke-specific factors that facilitate or misdirect effective communication between the caller and EMS. Further work is now underway to improve call handlers' recognition and response to calls in suspected acute stroke. The findings also underline the need for ongoing public education to raise public awareness of stroke symptoms and the appropriate response, in order to reduce delays in accessing emergency treatment.

\section{LIMITATIONS}

The study did not set out to identify and measure time delays between onset of symptoms and making an EMS call, as it was intended instead to study the caller's experience of the process of seeking emergency help in acute stroke. Although participants were interviewed soon after the event, the effects of recall bias may have influenced the findings.

Participants were representative in terms of the age and sex distribution of people who call 999 for suspected stroke. However, it was particularly difficult to contact and recruit callers who were unrelated to the patient, and this group is likely to be under-represented.

The study was concerned only with patients who accessed EMS via a 999 call in response to stroke symptoms. Further work would be needed to examine the experiences of patients who accessed healthcare by other routes such as direct presentation to the emergency department, who accessed other primary care services, or who did not seek health advice.

\section{SUMMARY}

At the onset of stroke, the caller's ability to convey their suspected diagnosis is limited by a lack of stroke symptom recognition, often as a result of the diverse presentation of stroke symptoms. Many callers believe that the onset of acute stroke symptoms does not necessarily warrant an immediate call to EMS. Delays often arise because the caller feels it necessary to seek other lay or professional advice prior to calling EMS.

Even when callers recognise stroke and call EMS, often they do not convey this impression to the call handler. The form of structured questioning used by the call handler does not always enable the caller to convey their full impression of what the problem is, or allow the call handler to assure callers that an ambulance has been arranged. In order to improve outcomes in hyper-acute stroke care, more public education is needed to 
improve awareness of a wider range of stroke symptoms and the need for an urgent response.

Funding This study was funded by the National Institute for Health Research (ref: RP-PG-0606-1066). The views and opinions expressed within this paper do not necessarily reflect those of the NIHR.

\section{Competing interests None.}

Ethics approval This study was conducted with the approval of the Cumbria \& Lancashire B Research Ethics Committee.

Contributors SPJ: design and methodology, data collection, data analysis, full checking of final draft. HAD: data collection, data analysis, full checking of final draft. GAF: full checking of final draft. JMEG: data analysis, full checking of final draft. MJL: design and methodology, full checking of final draft. JJMCA: data collection, data analysis, full checking of final draft. AMcL: data collection, data analysis, full checking of final draft. TQ: full checking of final draft. CLW: design and methodology, full checking of final draft.

Provenance and peer review Not commissioned; externally peer reviewed.

\section{REFERENCES}

1. Lopez AD, Mathers CD, Ezzati M, et al. Global and regional burden of disease and risk factors, 2001: systematic analysis of population health data. Lancet 2006;367:1747-57.

2. The European Stroke Organization (ESO) Executive Committee and the ESO Writing Committee, 2009). Guidelines for Management of Ischaemic Stroke and Transient Ischaemic Attack 2008. http://www.eso-stroke.org/recommendations.php (accessed 15 Jun 2010).
3. Barsan WG, Brott TG, Broderick JP, et al. Time of hospital presentation in patients with acute stroke. Arch Intern Med 1993;153:2558-61.

4. Bratina P, Greenberg L, Pasteur W, et al. Current emergency department management of stroke in Houston, Texas. Stroke 1995:26:409-14.

5. Zweifler RM, York D, Mendizabal TUJ, et al. Accuracy of paramedic diagnosis of stroke. J Stroke Cerebrovasc Dis 1998;7:446-8.

6. Deakin CD, Alasaad M, King P, et al. Is ambulance telephone triage using advanced medical priority dispatch protocols able to identify patients with acute stroke correctly? Emerg Med J 2009;26:442-5.

7. Rosamond WD, Evenson KR, Schroeder EB, et al. Calling emergency medical services for acute stroke: a study of 9-1-1 tapes. Prehosp Emerg Care 2005:9:19-23.

8. Patton M0. Qualitative Evaluation and Research Methods. 2nd edn. Newbury Park CA: Sage Publications, 1990

9. Glaser BG. Basics of Grounded Theory Analysis. Emergence vs Forcing. Mill Valley, CA: Sociology Press, 1992

10. Porteous GH, Corry MD, Smith WS. Emergency medical services call handle identification of stroke and transient ischemic attack. Prehosp Emerg Care 1999:3:211-16.

11. Mosley I, Nicol M, Donnan G, et al. Stroke symptoms and the decision to call for an ambulance. Stroke 2007;38:361-6.

12. Carroll C, Hobart J, Fox C, et al. Stroke in Devon: knowledge was good, but action was poor. J Neurol Neurosurg Psychiatry 2004;75:567-71.

13. Pattenden J, Watt I, Lewin RJ, et al. Decision making processes in people with symptoms of acute myocardial infarction: qualitative study. BMJ 2002:324:1006-11.

14. Jones SP, Jenkinson AJ, Leathley MJ, et al. Stroke knowledge and Public awareness: an integrative review of the evidence. Age Ageing 2010;39:11-22. 